\title{
47.2: Hole Injection and Power Efficiency of Organic Light Emitting Diodes with Ultra-Thin Inorganic Buffer Layer on Indium Tin Oxide
}

\author{
Chengfeng Qiu, Haiying Chen, Zhiliang Xie, Man Wong and Hoi-Sing Kwok \\ Center for Display Research, Department of Electrical and Electronic Engineering \\ The Hong Kong University of Science and Technology \\ Clear Water Bay, Kowloon, Hong Kong
}

\begin{abstract}
ITO capped with a variety of ultra-thin metal layers such as platinum, manganese, nickel, gold, lead, magnesium, and nonmetal layer such as, carbon, gallium, silicon, has been used as hole-injecting anode in organic light-emitting diodes consisting of $\mathrm{CuPc} / \mathrm{TPD} / \mathrm{Alq}_{3}$. Enhancement in hole injection but not current nor power efficiencies have been obtained in devices with metalcapped ITO, regardless of the work-function of the metals. For devices with silicon-capped anodes, improvements in hole injection, current and power efficiencies have been obtained.
\end{abstract}

\section{Introduction}

Organic light-emitting diode (OLED) is challenging liquidcrystal (LC) as an alternative flat-panel display technology because of its ease of manufacturing, all solid-state design, faster switching speed and being self-emitting with a wider viewing angle [1].

The performance of OLEDs is influenced not only by the properties of their constituent organic materials, but also by the electrodes and the interfaces they form with the carrier transport layers. Electro-luminescence (EL) efficiencies in OLEDs can be improved by enhancing either the internal quantum efficiency [2] or the injection efficiencies of electrons $[3,4]$ or holes [5]. The latter typically results in a reduction of the OLED driving voltage [6].

For the injection of electrons, metals with low workfunctions [3] are typically used as cathodes. Such metals tend to be highly reactive, hence unstable. They are typically capped with more stable metals. Examples of composite cathodes are $\mathrm{Ca} / \mathrm{Al}$, $\mathrm{Mg} / \mathrm{Ag}, \mathrm{Mg} / \mathrm{MgAg}$ and $\mathrm{Gd} / \mathrm{Al}$ etc [7-9]. Alternatively, ultra-thin insulating compounds containing low work-function metals have been used to generate interfacial dipoles that result in favorable alignments of the Fermi levels of the cathode and the lowestunoccupied molecular orbital (LUMO) energy levels of the electron-transport layers. The barrier against electron injection is subsequently reduced. Examples of such electron injection promoter are $\mathrm{LiF}$ and $\mathrm{CsF}$. [10,11]

For the injection of holes, indium-tin oxide (ITO) has been popularly used as a transparent anode. However, ITO needs to be properly treated for efficient hole injection. Such surface treatments include ultra-violet ozone cleaning [12], argon ion bombardment [13] or oxygen plasma exposure [14]. In addition, organic anode buffer layers, such as copper (II) phthalocyanine $(\mathrm{CuPc}),[15]$ with suitable highest-occupied molecular orbital (HOMO) energy levels or metals with high work-functions, such as $\mathrm{Au}, \mathrm{Ni}$ and $\mathrm{Pt}$ [16] can be used to enhance hole injection efficiencies.

Surface treatments of ITO increases not only the hole injection efficiency, but also the quantum and power efficiencies.
However, for metal buffer layers, improvement was observed only in hole injection efficiency, but not in power efficiency [16]. This clearly implies reduced quantum efficiency, which is undesirable. Furthermore, such metals are only semi-transparent, hence diminishing potential gains in efficiencies resulting from the metal buffer layer.

In this paper, we systematically investigated hole injection and power efficiency for the OLEDs containing ultra-thin $(1 \mathrm{~nm})$ layers of metals or non-metal layer on ITO. Most high work function metal layers can improved the hole-injection efficiency, but not the power efficiencies. It was found that a Si layer can enhanced both the hole injection and power efficiencies of the OLED.

\section{OLED fabrication}

The starting substrates were commercial glass coated with $70 \mathrm{~nm}$ ITO having a sheet resistance of $30 \Omega$. The sequence of precleaning prior to loading into the evaporation chamber consisted of ultra-sonic detergent soak for 30mins, de-ionized (DI) water spray for $10 \mathrm{mins}$, ultra-sonic DI water soak for $30 \mathrm{mins}$, oven bake-dry for 1-2hrs and UV ozone illumination for 9mins [17].

The ultra-thin capping elements were evaporated using 99.99\% pure powder loaded in resistively heated evaporation cells. The range of the deposition rates of the various elements was $0.01-0.03 \mathrm{~nm} / \mathrm{s}$. After the evaporation, the samples were subjected again to DI water rinse and UV ozone exposure. It is expected that some of the more reactive elements are oxidized during this treatment.

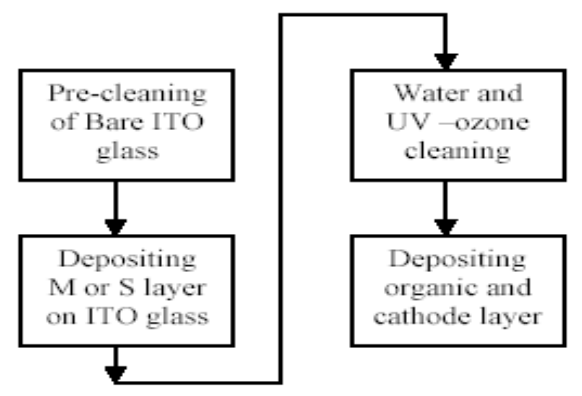

Figure 1. The fabrication process of the OLEDs

The constituent organic layers for the OLEDs were next deposited on the substrates using thermal vacuum evaporation of commercial grade Copper phthalocyanine (CuPc), N, N'diphenyl-N,N' bis(3-methylphenyl-1,1'-biphenyl-4,4'-diamine (TPD) and tris-8-hydroxyquinoline aluminum $\left(\mathrm{Alq}_{3}\right)$ powder sources. The base pressure in the evaporator was $\sim 8 \mu$ Torr. The deposition rates of the organic thin films were $0.2-0.4 \mathrm{~nm} / \mathrm{s}$. While 
ITO with or without capped layers formed the anodes of the OLEDs, $0.1 \mathrm{~nm}$ lithium fluoride (LiF) topped with $150 \mathrm{~nm}$ aluminum (Al) composite layers were used as the cathodes. The deposition rates of $\mathrm{LiF}$ and $\mathrm{Al}$ were $0.02-0.05 \mathrm{~nm} / \mathrm{s}$ and $1-1.5 \mathrm{~nm} / \mathrm{s}$, respectively. Film thickness was determined in situ using a crystal monitor. The fabrication process of the OLEDs are summarized in Figure 1.

Three types of $4 \mathrm{~mm}$-diameter OLEDs (Fig. 2) were fabricated using a set of shadow masks. These are:

1. Device C: $\operatorname{ITO}(75 \mathrm{~nm}) / \mathrm{CuPc}(20 \mathrm{~nm}) / \mathrm{TPD}(40 \mathrm{~nm}) / \mathrm{Alq}_{3}$ $(50 \mathrm{~nm}) / \mathrm{LiF}(1 \mathrm{~nm}) / \mathrm{Al}(150 \mathrm{~nm})$,

2. Device M: ITO/M $(1 \mathrm{~nm}) / \mathrm{CuPc} / \mathrm{TPD} / \mathrm{Alq}_{3} / \mathrm{LiF} / \mathrm{Al}$, $\mathrm{M}: \mathrm{Pt}, \mathrm{Mn}, \mathrm{Au}, \mathrm{Ni}, \mathrm{Pb}$ or $\mathrm{Mg}$.

3. Device S: $\mathrm{ITO} / \mathrm{S}(1 \mathrm{~nm}) / \mathrm{CuPc} / \mathrm{TPD} / \mathrm{Alq}_{3} / \mathrm{LiF} / \mathrm{Al}$. $\mathrm{S}: \mathrm{C}, \mathrm{Si}$ or $\mathrm{Ga}$.

Against the performance of the control device Type C, that of device Types $\mathrm{M}$ and $\mathrm{S}$ was compared.

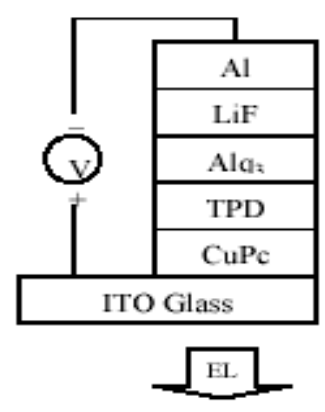

Device $\mathrm{C}$

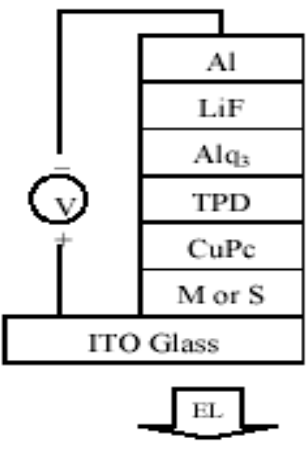

Device $\mathrm{M}$ or $\mathrm{S}$
Figure 2. Structure of the OLEDs

\section{Results:}

\subsection{Metal layer in device}

Figure 3 and Figure 4 show the current density and luminance as a function of operating voltage for a set of device, device-C with bare ITO, and device-M with $\mathrm{Pt}, \mathrm{Mn}, \mathrm{Au}, \mathrm{Ni}, \mathrm{Pb}$, $\mathrm{Mg}$ layers capped on ITO, respectively. As apparent from the results, significant change in current density-voltage curve $(\mathrm{J}-\mathrm{V})$ and luminance-voltage (L-V) characteristics in devices with metal layer capped. Clearly, by capping ultra-thin metal layer on ITO, Both J-V curve and L-V curve shift to the lower voltage. Generally, devices-M with high work function metal has high current density and high luminance at the same voltage, except for Mn. Figure 4 gives the similar result as in Figure 3, the higher work-function of the metal will decrease the operating voltage more at the constant luminance, except for Mn.

The inset of Figure 3 shows the change of voltage with the work function of the insert metal of the device-M at the $100 \mathrm{~A} / \mathrm{m} 2$ current density. The work functions of the metals in inset of figure 3 come from reference [18]. It can be seen from the inset of Fig.3, the higher of the work function of the metal is, the lower of operating voltage of the devices will be.

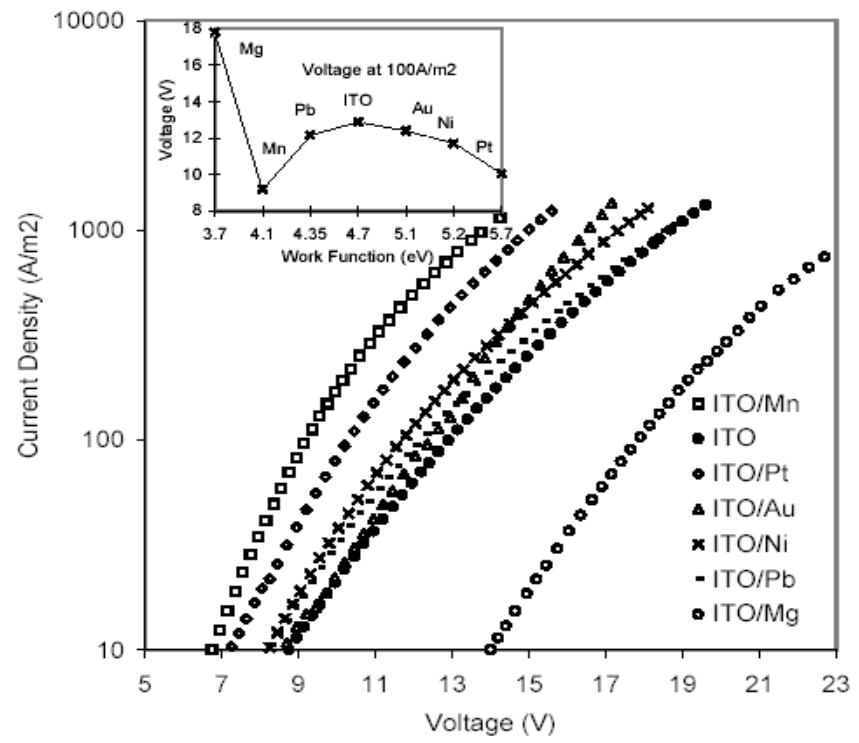

Figure 3. Current density-Voltage curve of Device-M

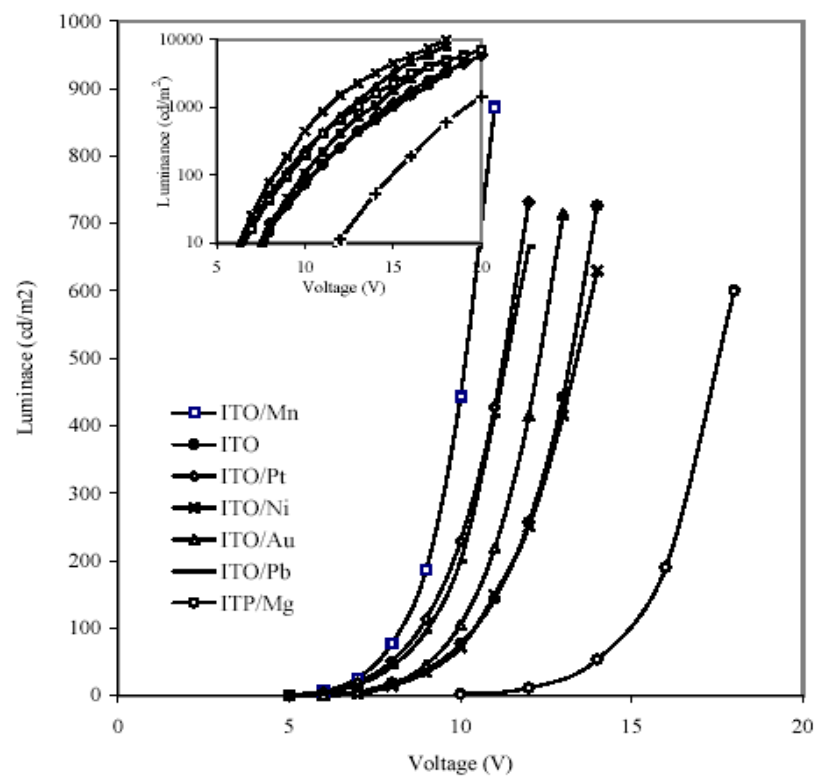

Figure 4. Luminance-Voltage curve of Device-M

According to the formula of power efficiency [19],

$\eta=\frac{\pi L}{V J}=\pi \frac{1}{V}\left(\frac{L}{J}\right) \equiv \pi \frac{\gamma}{V}$

where $\eta$ is the power efficiency and $\gamma$ is the current efficiency of the device. We can guess that if insertions of ultra-thin metal layer in the device do not affect the quantum efficiency, the power efficiency the device-M will increase with the high work-function of the metal.

Table I give the power efficiency and current efficiency of device-M. It can be seen that all the metals decrease the current efficiency of the OLEDs. The power efficiency is also decreased, 
except for $\mathrm{Pt}$ and $\mathrm{Mn}$. Because $\mathrm{Pt}$ and $\mathrm{Au}$ enhance the hole injection more, according to eq. (1), the enhanced hole injection makes up for the decrease of the current efficiency, so they do not effect the power efficiency of the device [16] on appearance.

Table 1. Summary of the performance of OLEDs with ITO capped with a variety of metals and non-metals

\begin{tabular}{|c|c|c|c|c|c|c|c|c|c|}
\hline & Type C & \multicolumn{5}{|c|}{ Type M } & \multicolumn{4}{|c|}{ Type S } \\
\hline Inserted material & & $\mathrm{Mn}$ & $\mathrm{Pt}$ & $\mathrm{Ni}$ & $\mathrm{Au}$ & $\mathrm{Pb}$ & $\mathrm{Ga}$ & $\mathrm{C}$ & $\mathrm{Si}$ \\
\hline $\begin{array}{c}\text { Power Efficiency } \\
\text { (Im/w) }\end{array}$ & 1.0 & 0.9 & 0.9 & 0.6 & 0.7 & 0.9 & 1 & 0.7 & 1.2 \\
\hline $\begin{array}{c}\text { Current Efficiency } \\
\text { (cd/A) }\end{array}$ & 3.5 & 2.7 & 2.7 & 2.4 & 2.5 & 3.3 & 3.4 & 2.0 & 3.51 \\
\hline
\end{tabular}

\subsection{Non-metal layer in device}

Figure 5 and Figure 6 show the current density and luminance as a function of operating voltage for a set of device, device-C with bare ITO, and device-S with $\mathrm{C}, \mathrm{Si}$, Ga layers on ITO, respectively. As apparent from these figures, similar results as devices-M are obtained. Higher work function of the inserted layer show higher current density and luminance at the same operating voltage. So silicon, carbon and gallium all enhance the hole injection of the devices-S. From Figure 5, it can be seen that device-C has higher current density than that of device-Si.

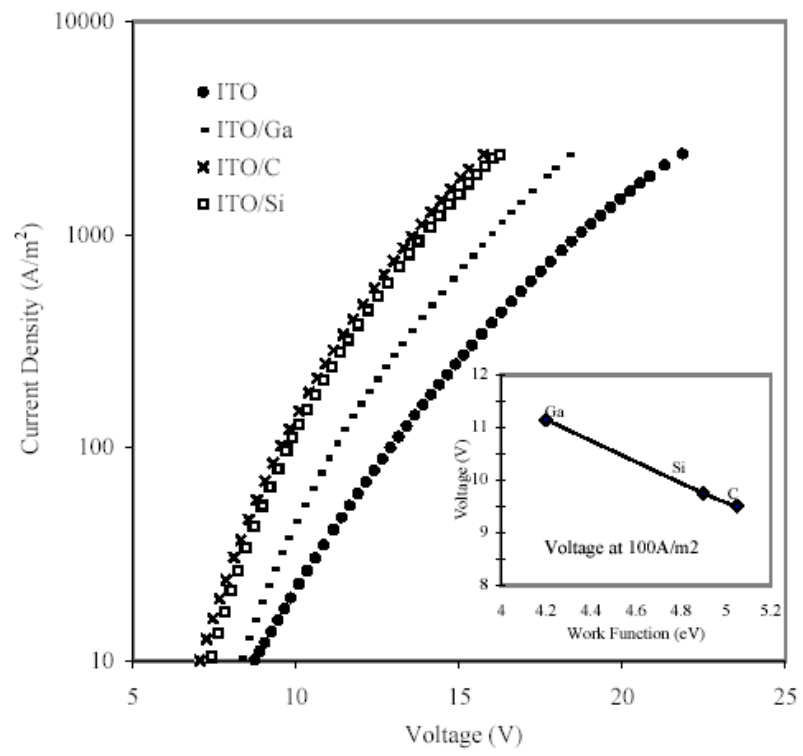

Figure 5. Current density-Voltage curve of Device-S

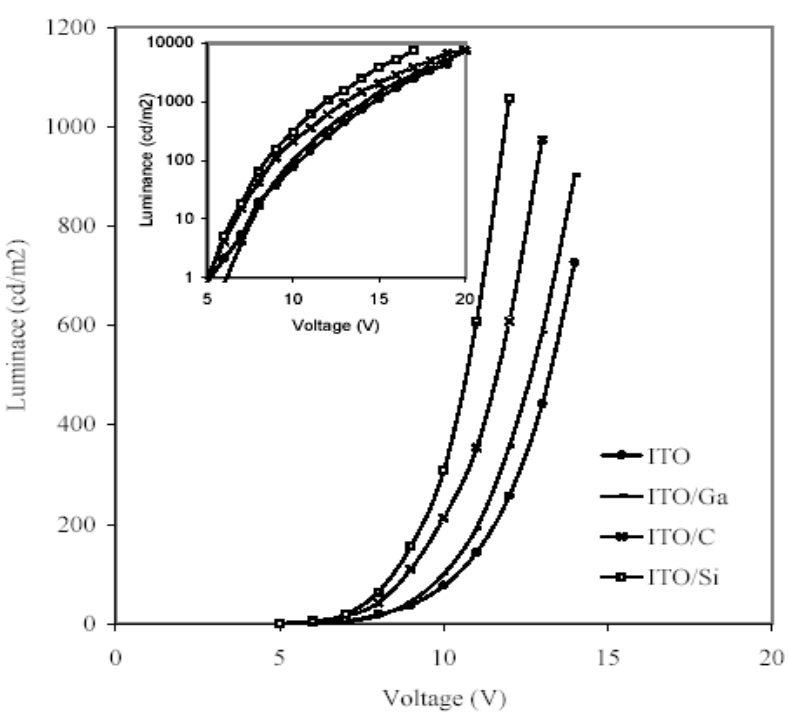

Figure 6. Luminance-Voltage curve of Device-S

In Figure 6, the L-V curve of the device with $\mathrm{C}$ on ITO and device with $\mathrm{Si}$ on ITO are reversed to Figure 5. This implies that device with $\mathrm{C}$ on ITO has lower EL current efficiency than the device with Si on ITO. This can be seen in Table I as well. The EL current efficiency of device with $\mathrm{C}$ on ITO is only $2 \mathrm{Cd} / \mathrm{A}$, but the EL current efficiency of the device with $\mathrm{Si}$ on ITO is $3.52 \mathrm{Cd} / \mathrm{A}$, almost same as that of device-C.

Figure 7 give the EL power efficiency of the device-S, we can see that the device with $\mathrm{Si}$ on ITO has higher power efficiency. So Si on ITO enhances the hole injection efficiency and improves the power efficiency at the same time.

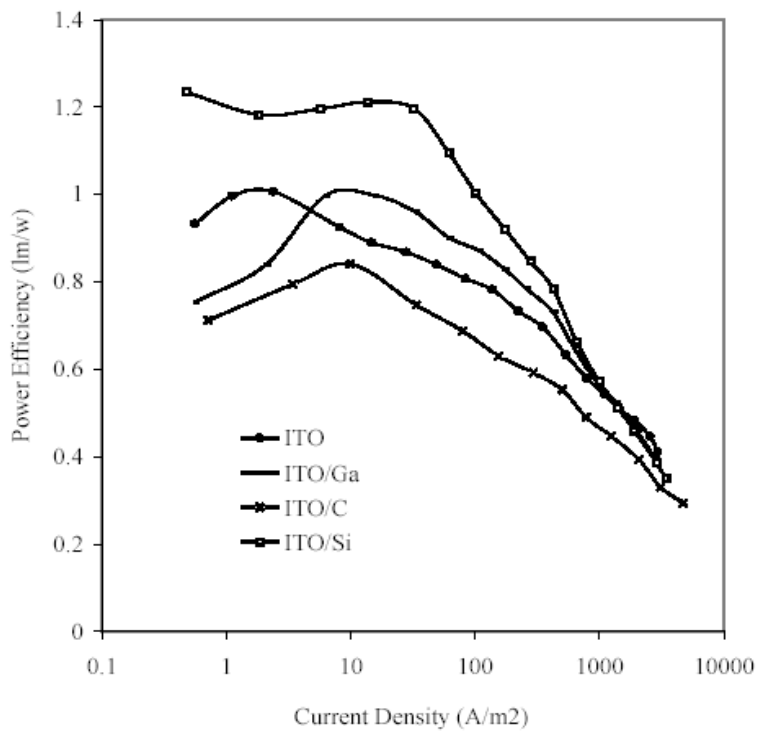

Figure 7. Power efficiency-Current density curve of Device-S

\section{Discussions}

From the above results, it is obvious that the best buffer layer is $\mathrm{Si}$. In order to examine the physical mechanism for the improvements, we performed an XPS study of the Si buffer layer. 
Fig. 8 shows the results. It is confirmed that indeed the Si layer is oxidized to $\mathrm{SiO}_{2}$ after the ozone treatment. In fact, even without the ozone treatment, the ultra-thin Si layer will simply oxidize in air to become $\mathrm{SiO}_{2}$.

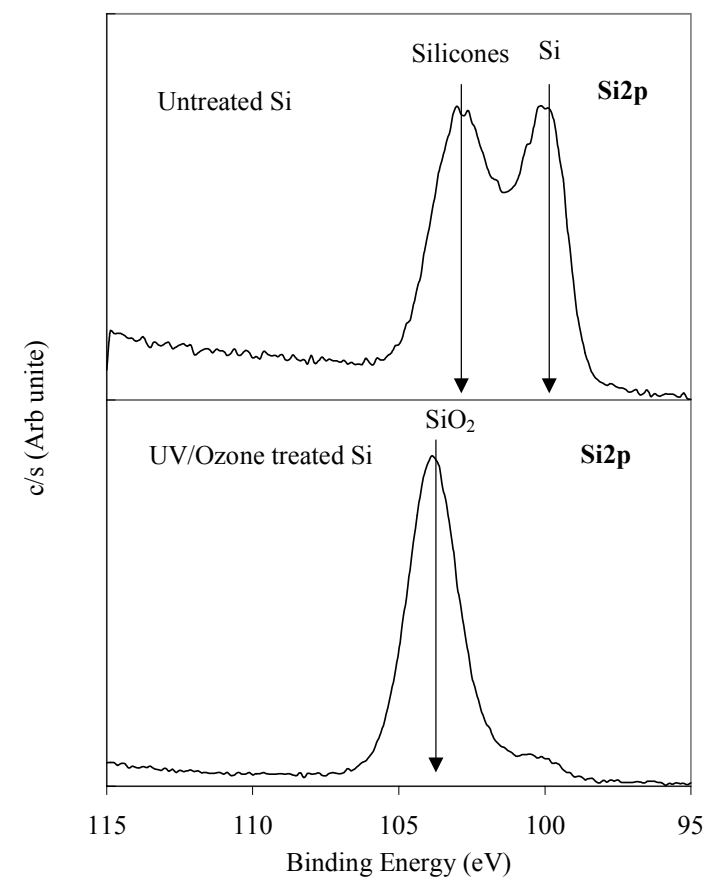

Figure 8. XPS spectra of the Si layer before and after treatment.

The $\mathrm{SiO}_{2}$ layer is insulating. It is therefore believed that the physical mechanism for the improve power and quantum efficiencies is similar to the insulating $\mathrm{LiF}$ layer for $\mathrm{Al}$ cathode [17]. The insulating layer provides dipoles for band alignment.

\section{Conclusions}

The insertion of a series of ultra-thin metal or non-metal layer between ITO and HTL was investigated systematically for the first time. We found that an ultra-thin metal layer on ITO can enhance the hole injection efficiency, but cannot improve the power efficiency of the OLEDs. A Si layer on ITO can enhance both the hole injection the power efficiency. But the current efficiency is not enhanced. Finally, the $\mathrm{Si}$ layer actually is a $\mathrm{SiO}_{2}$ layer due to oxidation.

\section{Acknowledgements}

This work was supported by the Research Grants Council of the Hong Kong Special Administrative Region.

\section{References}

[1]. C. W. Tang and S. A. VanSlyke, Appl. Phys. Lett. Vol.51,pp913-915,(1987)

[2]. Masamichi Ikai, Shizuo Tokito, and Youichi Sakamoto, Toshiyasu Suzuki, and Yasunori Taga, Appl. Phys. Lett. Vol.79 pp156-158(2001)

[3]. M. Stossel, J. Staudigel, F. Steuber, J. Simmer, A. Winnacke, Appl. Phys. A vol.68, pp387-390(1999)

[4]. T. M. Brown and R. H. Friend, I. S. Millard, P. J. Lacey and J. H. Burroughes, F. Cacialli, Appl. Phys. Lett vol.79p174-176(2001)

[5]. Beomrak Choi, Hyunsik and Hong H. Lee, Appl. Phys. Lett. Vol.76 pp412-414(2000)

[6]. X. Zhou, M. Pfeiffer, J. Blochwitz, A. Werner, A. Nollau, T. Fritz, and K. Leo, Appl. Phys. Lett. Vol.78 pp410412(2001)

[7]. Jayesh M. Bharathan and Yang Yang, J. Appl. Phys. Vol.84(6) pp 3207-3211, (1998)

[8]. M. Stoßel, J. Standigel, F. Steuber, J. Blässing, J. Simmerer, A. Winnacker, H. Neuner, D. Metzdorf, H-H. T.Johannes, W. Kowalsky, Synthetic Metals 111112(2000), p19-24

[9]. Michio Matsumura, Atsushi Ito and Yuichiro Miyamae, Appl. Phys. Lett. Vol.75 pp1042-1044(1999)

[10]. L. S. Hung, C. W. Tang, and M. G. Mason, Appl. Phys. Lett. 70(2), pp152-154(1997)

[11]. Pongpun Piromreun, HwanSool Oh, Yulong Shen, and George G. Malliaras, J. Campbell Scott and Phil J. Brock, Appl. Phys. Lett. 77(15), pp 2403-2405(2000)

[12]. C. C. Wu, C. I. Wu, J. C. Sturm, and Kahn, Appl. Phys. Lett. Vol.70 pp 1348-1350(1997)

[13]. J. S. Kim, M. Granström, and R. H. Friend, N. Johansson and W. R. Salaneck, R. Daik and W. J. Feast, and F. Cacialli, J. Appl. Phys. Vol.84(12),pp6859-6870(1998)

[14]. M. G. Mason, L. S. Hung, and C. W. Tang, S. T. Lee, K. W. Wong, and M. Wang, J. Appl. Phys. Vol. 86(3), pp1688-1692(1999)

[15]. S. A. Van Slyke, C. H. Chen and C. W. Tang Appl. Phys. Lett., Vol.69(15), (1996), pp2160-2162

[16]. Yulong Shen, Daniel B. Jacobs, George G. Malliaras, Goutam Koley, Michael G. Spencer, and Andronique Ioannidis, Adv. Mater. Vol.13(16) pp1234-1238(2001)

[17]. C. Qiu, H. Chen, M. Wong and H. S. Kwok, IEEE Trans. on Electron Devices, Vol.48(9), (2001), pp2131-2137.

[18]. S.M. Sze, Physics of Semiconductor Devices (Wiley, New York 1981)

[19]. J.-S. Kim, P. K. H. Ho, N. C. Greenham, and R. H. Friend, J. Appl. Phys. Vol. 88(2), pp1073-1081(2000) 